\title{
Anaemia - a common syndrome in old age
}

\begin{abstract}
Anaemia is a common syndrome in the elderly (age $>65$ years), combined with changes and diseases characteristic of ageing. There are currently nearly 500 million (7\%) adults over the age of 65 in the world. According to statistics, there are about 15 million older people with anaemia in the European Union. This number is likely to increase in the coming years due to the ageing population in Western societies. ${ }^{1}$ The acute anaemic syndrome is dominated by symptoms of decreased circulatory volume, such as dizziness, syncope and hypotension. While in the chronic course, anaemia can be asymptomatic and be detected accidentally in a laboratory test. Suspicious signs are a reduced ability to carry oxygen, such as general weakness, fatigue and shortness of breath, for which age changes are often blamed. Worsening of concomitant conditions such as angina, heart failure, CKD and chronic obstructive pulmonary disease has been observed. Older people with anaemia of any degree have a deteriorating quality of life due to significantly higher morbidity and mortality. The purpose of this review is to summarize the most common etiological causes of anaemia in the elderly, clinical consequences, and guidelines for diagnosis.
\end{abstract}

Volume 6 Issue 4 - 2021

\section{Mariana Georgieva Yordanova}

Department of Clinical Laboratory, Military Medical Academy, Multiprofile Hospital for Active Treatment-Varna, Bulgaria

\begin{abstract}
Correspondence: Mariana Georgieva Yordanova, Department of Clinical Laboratory, Military Medical Academy, Multiprofile Hospital for Active Treatment, Medical University of Varna, Bulgaria,Tel +359882799904, Email m.jordanova.doc@abv.bg
\end{abstract}

Received: December 01, 2021 | Published: December 16, 2021

\section{Introduction}

Modern health and social care face growing challenges for a rapidly ageing population due to the significant advances in public health, medical and pharmacological research and preventive medicine. People over the age of 80 are the fastest-growing part of the population and are expected to reach nearly $30 \%$ of the total population in Western societies by the 2050 year. $^{2}$ Ageing is usually accompanied by chronic (multiple) diseases, disability, weakness and social isolation. ${ }^{3}$ Anaemia is a common syndrome in the elderly (age $>65$ years), but its spread should not be considered an inevitable ageing consequence. Over the last decade, it has emerged as a significant risk factor associated with various adverse outcomes in the elderly, including increased hospitalization, disability and mortality. ${ }^{4}$

\section{Defining anaemia}

Anaemia is defined as a decrease in haemoglobin and/or erythrocytes and hematocrit below the lower reference limit for healthy individuals, necessarily consistent with gender, age, race, altitude, etc., environmental factors. The overall incidence of anaemia in the elderly is about $12-17 \%$, reaching $47 \%$ in those living in nursing homes and up to $40 \%$ in hospitalized patients. ${ }^{5}$ Its prevalence can get nearly $50 \%$ in men over 80 in both hospital and outpatient patients. Anaemia is essentially a homeostatic imbalance in the concentration of haemoglobin in the blood, in which the destruction or loss of erythrocytes is more significant than their production. ${ }^{4}$ According to WHO criteria for anaemia, haemoglobin $(\mathrm{Hb})$ values are below $130 \mathrm{~g} / \mathrm{L}$ in men, below $120 \mathrm{~g} / \mathrm{L}$ in women and less than $110 \mathrm{~g} / \mathrm{L}$ in pregnant women and children. For the elderly, haemoglobin values have different reference limits that do not meet the above criteria. However, most clinicians accept the WHO definition and believe that the normal range of haemoglobin in the elderly should not be adjusted due to its association with morbidity, mortality and hospitalizations. ${ }^{6}$

Reference limits for normal haemoglobin vary from laboratory to laboratory. They usually depend on the method of determination, the automatic cell counter and the reagents used by a laboratory. Most guidance in clinical practice is to determine the haemoglobin reference limits according to age and sex for a given population group associated with a particular lifestyle and culture. It is difficult to find a reference group of "healthy" adult subjects due to the high percentage of comorbidities and disabilities with age. For example, in the analysis of Cheng et al. ${ }^{7} 60 \%$ of the elderly were excluded from the reference group due to common diseases, including obesity, hypertension, diabetes, recent treatment of anaemia or recent surgery or hospitalization. The introduction of this approach limits its practical applicability. Another approach is based on determining optimal $\mathrm{Hb}$ concentrations for the clinical course and with minimal risk of adverse outcomes in the elderly. A study in an adult group for cardiovascular health assessment found increased mortality in the lower quintile for haemoglobin $<137 \mathrm{~g} / \mathrm{L}$ for men and $<126 \mathrm{~g} / \mathrm{L}$ for women was found. ${ }^{8}$ Several studies have shown that anaemia correlates with increased hospitalization ${ }^{9}$ and poorer survival in the elderly. ${ }^{10}$ For example, mild anaemia or the so-called "low-normal" haemoglobin level is associated with a wide range of more adverse health problems. For example, patients with heart failure with haemoglobin levels in the lowest quartile have more symptoms, poorer hemodynamics, and higher mortality than those with higher haemoglobin levels. ${ }^{11}$ The proposed optimal $\mathrm{Hb}$ value to avoid hospitalization and mortality is $130-150 \mathrm{~g} / \mathrm{L}$ for women and $140-170 \mathrm{~g} / \mathrm{L}$ for men, suggesting a redefinition of the limit values for anaemia. ${ }^{6}$

\section{Diagnostic aspects}

Anaemia is a multifactorial condition that accompanies increased comorbidity in the elderly. Diagnosis and treatment in this age group often require a multidisciplinary approach and detailed studies of the functional state of the organs. ${ }^{1}$ The most common causes of anaemia in the elderly include reduced nutrition with nutritional deficiencies, chronic inflammatory diseases, chronic renal failure, liver disease, occult blood loss in degenerative and malignant gastrointestinal tract diseases, and suppression of hematopoiesis (decline in proliferation stem cells from drugs, alcohol use, chronic diseases, myelodys plastic syndrome (MDS), etc.). However, in many patients, the aetiology may remain unknown. ${ }^{1}$ History and clinical status should focus on identifying risk factors and symptoms specific to a specific condition. For example, melena, hematochezia, and weight loss may indicate neoplastic gastrointestinal bleeding. Primary laboratory evaluation in elderly patients with anaemia should include basic parameters, including $\mathrm{Hb}$, differential blood count, mean corpuscular volume $(\mathrm{MCV})$, mean corpuscular haemoglobin $(\mathrm{MCH})$, reticulocyte count, ferritin, erythropoietin level (EPO), C -reactive protein (CRP), 
creatinine, filtration rate (EGF), serum folate, lactate dehydrogenase (LDH), haptoglobin.

\section{Erythrocyte indices}

Peripheral blood count $(\mathrm{CBC})$ is the most routine diagnostic procedure. When anaemia is detected, the main goal is to differentiate its morphological type. Based on erythrocyte indices (MCV) and mean erythrocyte haemoglobin $(\mathrm{MCH})$, anaemia is classified as microcytic, hypochromic (MCV $<80 \mathrm{fL} ; \mathrm{MCH}<28 \mathrm{pg}$ ), normocytic, normochromic (MCV 80 $\div 100 \mathrm{fL}$; MCH $28 \div 32 \mathrm{pg}$ ) and macrocytic, hyperchromic (MCV> $100 \mathrm{fL} \mathrm{MCH}>32 \mathrm{pg}$ ). Red blood cell distribution width (RDW 11.5 $\div 14.5 \%$ ) is another parameter that gives a digital expression of the concept of anisocytosis, a characteristic of anaemia. It can detect iron deficiency early before changes in MCV. These haematological indicators allow a specific and personalized anaemia assessment and set the direction for additional biochemical and haematological studies to clarify its aetiology.

"Who diagnoses well, that well-treat" Hippocrates

\section{Characterization of anaemia in the elderly}

The most common morphological types in the elderly are microcytic and normocytic anaemia. Although microcytic anaemia is classically associated with iron deficiency, normocytic anaemia with chronic disease has a significant overlap between the manifestations of these diseases with age. ${ }^{12}$ Iron deficiency anaemia (IDA) is the most common anaemia in the elderly $\approx 25.3 \% .^{13}$ It occurs in $11 \%$ to $57 \%$ of patients with colorectal cancer and may be an initial symptom in approximately $15 \% .{ }^{14}$ Therefore, endoscopic examination is necessary to assess the gastrointestinal tract in search of degenerative or malignant diseases that cause nutritional deficiency from malabsorption or chronic blood loss. Annual examination of occult blood in faecal mass in adult patients is a perfect screening approach for early detection of gastrointestinal blood loss. Laboratory parameters that are reasonable to test in patients with this morphological type of anaemia are serum iron, total iron-binding capacity, transferrin saturation and ferritin. However, a study of hospitalized patients over 80 showed that these routine blood tests had poor screening sensitivity to capture iron-deficient patients. ${ }^{4,15}$

Patients with normocytic normochromic anaemia may have normal or low iron levels and standard or even high ferritin levels. This constellation, combined with elevated levels of the inflammatory marker C-reactive protein (CRP), is most common in chronic inflammatory (CID) and neoplastic (ND) diseases (outside the gastrointestinal tract). At least one-third of patients with anaemia over 65 show a condition typical of CKD or associated with infection (cancer, autoimmune disease, and chronic illness). The role of various cytokines, such as tumour necrosis factor $\alpha$ (TNF $\alpha$ ), interleukin-1 (IL-1) and transforming growth factor $\beta$ (TGF-Beta), is mentioned as part of the pathophysiological mechanisms in this type of anaemia. They exert a direct negative effect on the proliferation and differentiation of erythroid progenitor cells. ${ }^{1}$ Hepcidin is an acutephase protein produced by the liver and plays a central role in iron metabolism. It reduces the absorption of iron in the duodenum and the release of iron from macrophages. Elevated hepcidin levels have been reported in patients with neoplastic, autoimmune diseases and CKD. In addition, elevated values have been reported in the elderly, possibly due to age-related changes. ${ }^{16}$ At the same time, increased iron uptake and retention in the reticuloendothelial system leads to iron-poor erythropoiesis. Ferritin, in addition to reflecting the body's iron stores, is also a positive acute-phase protein and its increase is expected in anaemia in CID and ND. In addition to chronic diseases, iron deficiency may also be imposed in adult patients. ${ }^{17}$ Free transferrin receptors and their ratio to ferritin are valuable markers for distinguishing IDA from anaemia due to CID or ND. These parameters are also relevant to the choice of an appropriate therapeutic approach. In normocytic normochromic anaemia, it is reasonable to determine serum creatinine levels and erythropoietin (EPO). Assessment of renal function and determination of reduced glomerular filtration rate is part of the etiological specification of secondary anaemia in the course of renal failure. ${ }^{13}$ Various studies have shown that the risk of anaemia is increased at the threshold of renal function with GFR $<40 \mathrm{~mL} / \mathrm{min}^{4}{ }^{4}$

In macrocytic and hyperchromic anaemia, diseases leading to vitamin B12 or folate deficiency are suspected. A common cause of B12 deficiency is atrophic gastritis with decreased gastric acid secretion, or after resection of the stomach or areas of the intestine. Many diseases and multimorbidity in elderly patients inevitably lead to the use of many drugs with changes in the function of the gastrointestinal tract. Impaired absorption of vitamin B12 and folate are found when taking alcohol or medications prescribed for gastrointestinal reflux, ulcers, even diabetes. According to literature data, classical pernicious anaemia with vitamin B12 deficiency in the elderly is relatively rare. In contrast, Helicobacter pylori infections, acid-reducing agents or atrophic gastritis can cause hypochlorhydria, which more often leads to malabsorption syndrome of cobalamin from food. ${ }^{1,18}$ However, one-third of the elderly with anaemia did not have a cause for the anaemia present and were therefore classified as 'unexplained anaemia'. Probably a part of this type of anaemia is due to myelodysplastic syndrome (MDS). It is more common in older people than in young individuals. The syndrome is characterized by impaired normal blood cells production, peripheral cytopenia and blasts in the bone marrow due to a defect in stem cell development. Anaemia occurs when the red blood cell line is also affected and should be considered for diagnosis when it is accompanied by abnormalities in the number and distribution of white blood cells or platelets. ${ }^{4}$

\section{Clinical significance of anaemia in the elderly}

The ageing process involves a sequence of changes in biological, functional, psychological, and social parameters that vary depending on genetic factors, age-related vulnerabilities, and differences in organ function and reserves ${ }^{2}$ The symptoms associated with anaemia that share the elderly is easy fatigue, palpitations even with light exercise, drowsiness. Syncopal accidents due to cerebral hypoxia are not excluded. Occasionally there are complaints of dull pain in the heart due to increasing oxygen starvation of the myocardium. With increasing hypoxia and the presence of atheromatous plaques in the vessels, the likelihood of an older person having a heart attack or stroke and other severe disorders, increases. A recent large study of nearly 300,000 participants evaluated the effect of hemoglobin concentrations on cardiovascular disease and all-cause mortality, showing that men with $\mathrm{Hb}$ concentrations below the normal range had a higher risk of death while not observed in women. Therefore, anaemia in the elderly is a serious problem and requires adequate care. ${ }^{19,20}$ The American Society of Hematology and the National Institute on Aging organized joint symposia in 2004 and 2005 to address the problem and identify appropriate measures. ${ }^{21}$ Increasing data from the literature supports the claim that mild anaemia or "low normal" haemoglobin levels are associated with many adverse health effects, both in specific comorbidities and with increased mortality. ${ }^{22}$ The most pronounced is the negative impact of anaemia on the state of working capacity, physiology and functional independence on people over 65 years of age. Anaemia is associated with increased 
weakness, decreased cognitive function, risk of developing dementia, decreased mobility, increased risk of recurrent falls, lower bone density and skeletal muscle strength, and increased incidence of severe depression. ${ }^{1,13,23,24}$

In addition, it has been found that anaemia may be an indicator of a more severe course of comorbid conditions or a weaker response to current therapy. For example, the effectiveness of radiation therapy and chemotherapy depends on an adequate supply of oxygen from haemoglobin for optimal tumour cell killing. ${ }^{25}$

Cognitive functions are strongly associated with haemoglobin levels, regardless of the aetiology of anaemia. Even mild anaemia can significantly affect physical and cognitive abilities and quality of life. In prospective studies, anaemia was associated with a $41-61 \%$ increased risk of dementia in the elderly. ${ }^{26}$ Some experimental studies in humans have shown that iron deficiency can cause decreased neurotransmitter synthesis, impaired neuronal myelination, and damage to brain mitochondria, leading to cognitive impairment..$^{27,28,29}$

Recently, more and more data are being established on the relationship between hemoglobin and the risk of dementia and Alzheimer's disease (AD). In a population-based study by Wolters FJ et al., ${ }^{30}$ in Rotterdam for a period of 12.5 years on the relationship between anemia and dementia, the authors reported that cerebral micro bleeding was more common in anemia and hemoglobin levels correlated inversely $(\mathrm{p}<0.0001)$ with cerebral perfusion. ${ }^{30}$ Wolters FJ et al. ${ }^{30}$ show that anemia increases the risk of dementia by $34 \%$, and for $\mathrm{AD}$ by $41 \%$, probably by the mechanism of hypoperfusion and reduced oxygen supply to the brain.

Several extensive cohort studies have demonstrate that WHOdefined anaemia is associated with an increased risk of death in the elderly. For example, a 2016 prospective British study of 220 patients with a mean age of 83.6 years found that anaemia was associated with increased mortality, regardless of the etiological cause, one year after hospitalization. Another similar study of 3,758 adult patients found that new-onset anaemia with a decrease in haemoglobin of $10 \mathrm{~g} / \mathrm{L}$ was associated with increased mortality with risk factors of 1.39 at a $95 \%$ confidence interval. ${ }^{12}$ Most studies have observed an inverse J-relationship between haemoglobin levels and mortality with increased risk due to the severity of anaemia.,31 Given the comorbidities, it is difficult to determine whether anaemia is a marker of the severity of the underlying disease or a mediator in the causal pathway leading to side and adverse effects.

\section{Conclusion}

Anaemia should be taken seriously, given adequate attention in clinical practice, and not simply considered a "physiological" consequence of ageing. It is a common multifactorial condition among the elderly, which is associated with various adverse outcomes, such as impaired quality of life, increased hospitalization, and mortality. Today, anaemia is a clinical challenge in everyday practice due to the growing ageing of the population worldwide. In old age, one or more etiological causes of the anaemic syndrome are often found. Clarifying the basic mechanisms of anaemia will help for individualized treatment algorithms.

\section{Acknowledgments}

None.

\section{Conflicts of interest}

The author declares there is no conflict of interest.

\section{References}

1. Stauder R, ValentP, Theurl I. Anemiaatolderage: tiologies, clinical implications, and management. Blood. 2018;131(5):505-514.

2. NobiliA, GarattiniS, Mannucci PM. Multiple diseases and poly pharmacy in the elderly: challenges for the internist of the third millennium. $J$ Comorb. 2011;1:28-44.

3. Christensen K, Doblhammer G, Rau R, et al. Ageing populations: the challenges ahead. Lancet. 2009;374(9696):1196-2008.

4. Patel KV. Epidemiology of anemia in older adults. Semin Hematol. 2008;45(4):210-217.

5. Lanier JB, Park JJ, Callahan RC. Anemia in Older Adults. Am Fam Physician. 2018;98(7):437-442.

6. Stauder R, Thein SL. Anemia in the elderly: clinical implications and new therapeutic concepts. Haematologica. 2014;99(7):1127-1130.

7. Cheng CK, Chan J, Cembrowski GS, et al. Complete blood count reference interval diagrams derived from NHANES III: stratificationbyage, sex, andrace. Lab Hematol. 2004;10(1):42-53.

8. Zakai NA, Katz R, Hirsch C, et al. A Prospective Study of Anemia Status, Hemoglobin Concentration, and Mortality in an Elderly Cohort: The Cardiovascular Health Study. Arch Intern Med. 2005;165(19):2214-2220.

9. Vermeulen J, Neyens JC, van Rossum E, et al. Predicting ADL disability in community-dwelling elderly people using physical frailty indicators: a systematic review. BMC Geriatr. 2011;33:1471-2318.

10. Houwelingen AH, denElzen WP, Mooijaart SP, et al. Predictive value of a profile of routine blood measurements on mortality in older persons in the general population: the Leiden 85-plus Study. PloSOne. 2013;8(3):e58050.

11. David P Steensma, Ayalew Tefferi. Anemia in the Elderly: How Should We Define It, When Does It Matter, and What Can Be Done? Mayo Clin Proc. 2007;82(8):958-966.

12. Lanier JB, Park JJ, Callahan RC. Anemia in Older Adults. Am Fam Physician. 2018;98(7):437-442.

13. Goodnough LT, Schrier SL. Evaluation and management of anemia in the elderly. Am J Hematol. 2014;89(1):88-96.

14. Hamilton W, Lancashire R, Sharp D, et al. The importance of anaemia in diagnosing colorectal cancer: a case-control study using electronic primary care records. Br J Cancer. 2008;98:323-327.

15. Rimon E, Levy S, Sapir A, et al. Diagnosis of iron deficiency anemia in the elderly by transferr in receptor-ferrit in index. Arch Intern Med. 2002;162:445-449.

16. Nemeth E, GanzT. Anemia of inflammation. Hematol Oncol Clin North Am. 2014;28(4):671-681.

17. Cullis J. Anaemia of chronic disease. Clin Med (Lond). 2013;13(2):193196.

18. Loikas S, Koskinen P, Irjala K, et al. Vitamin B12 deficiency in the aged: a population-based study. Age Ageing. 2007;36(2):177-183.

19. Lee G, Choi S, Kim K, et al. Association of hemoglobin concentration and its change with cardiovascular and all-cause mortality. J Am Heart Assoc. 2018;7(3):e007723.

20. Smith DL. Anemia in the elderly. Am Fam Physician. 2000;62(7):15651572 .

21. Steensma DP, Tefferi A. Anemia in the elderly: how should we define it, when does it matter, and what can be done? Mayo Clin Proc. 2007;82(8):958-966.

22. Dharmarajan TS, Avula S, Norkus EP. Anemia increases risk for falls in hospitalized older adults: an evaluation off alls in 362 hospitalized, ambulatory, long-termcare, and community patients. J Am Med Dir Assoc. 2006; 7(5):287-293. 
23. Onder G, Penninx BW, Cesari M, et al. Anemia is associated with depression in older adults: results from the In CHIANTI study. $J$ Gerontol A Biol Sci Med Sci. 2005;60(9):1168-1172.

24. Beghé C, Wilson A, Ershler WB. Prevalence and outcomes of anemia in geriatrics: a systematic review of the literature. Am J Med. 2004;116:3-10.

25. Rockwell S, Dobrucki IT, Kim EY, et al. Hypoxia and radiation therapy: past history, ongoing research, and future promise. Curr $\mathrm{Mol} \mathrm{Med}$. 2009;9(4):442-458.

26. Agrawal S, Kumar S, Ingole V, et al. Does anemia affects cognitive functions in neurologically intact adult patients: Two year crosssectional study atruraltertiary care hospital. $J$ Family Med Prim Care. 2019;8(9):3005-3008.

27. Sunil K, Abhijit A, Jahanvi B, et al. Exploring the relationship between the platelet indices and psychosocial morbidity in elderly patients at a rural medical college hospital. Gerontol Geriatric Stud. 2018;3:255-264.
28. Hong CH, Falvey C, Harris TB, etal. Anemia and risk of dementia in older adults: findings from the Health ABC study. Neurology. 2013;81(6):528533.

29. Hare D, Ayton S, Bush A, et al. A delicate balance: iron metabolism and diseases of the brain. Front Aging Neurosci 2013;5:34.

30. Wolters FJ, Zonneveld HI, Licher S, etal. Hemoglobin and anemia in relation to dementia risk and accompanying changes on brain MRI. Neurology. 2019;93(9):e917-e926.

31. Patel KV, Harris TB, Faulhaber M, et al. Racial variation in the relationship of anemia with mortality and mobility disability among older adults. Blood. 2007;109:4663-4670. 